Research Paper

\title{
Prostate-selective $\alpha$ antagonists increase fracture risk in prostate cancer patients with and without a history of androgen deprivation therapy: a nationwide population-based study
}

\author{
Wei-Heng Kao ${ }^{1, *}$, Chang-Fu Kuo ${ }^{2,3, *}$, I-Jun Chou ${ }^{4}$, Lai-Chu See ${ }^{2,5,6}$, Wen-Kuan \\ Huang', Meng-Jiun Chiou ${ }^{2}$, Weiya Zhang ${ }^{3}$, Michael Doherty ${ }^{3}$, Chun-Chieh Wang ${ }^{1,9}$, \\ Jun-Te Hsu ${ }^{8}$, Hsien-Hsin Chen ${ }^{1}$ and Ji-Hong Hong ${ }^{1,9,10}$

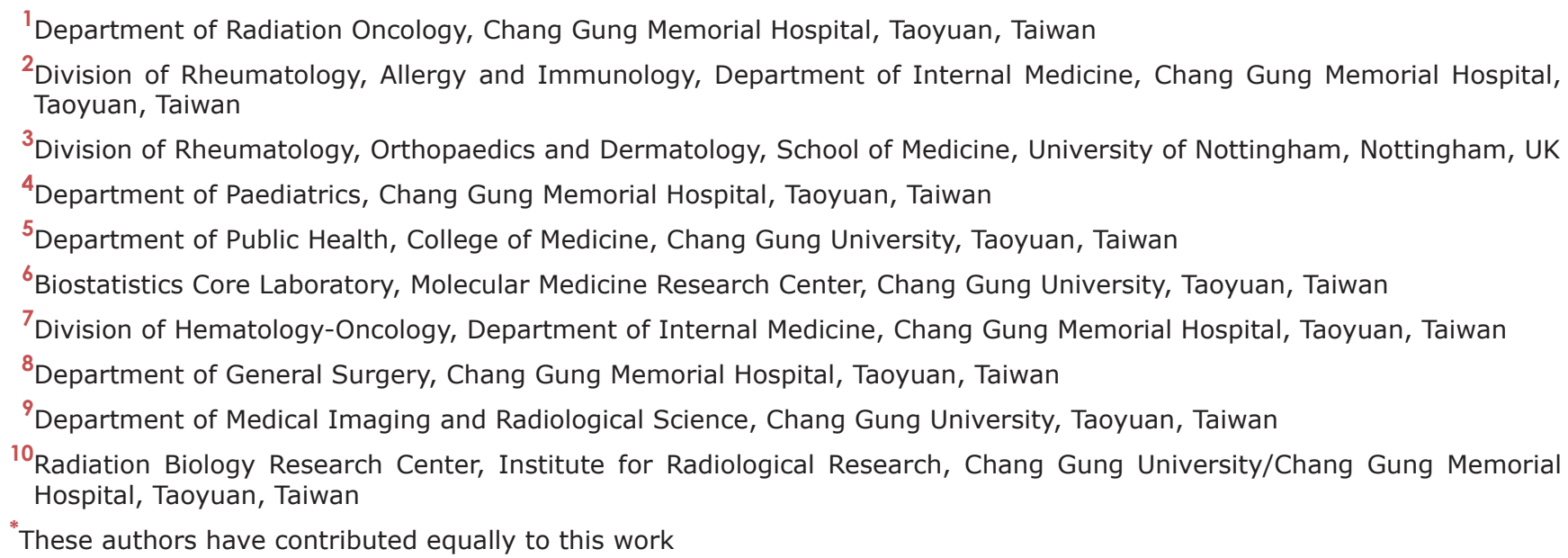

These authors have contributed equally to this work \\ Correspondence to: Ji-Hong Hong, email: jihong@cgmh.org.tw, fructose1202@gmail.com \\ Keywords: prostate-selective $\alpha$ antagonists; prostate cancer; androgen deprivation therapy; fracture; population-based study \\ Received: May 13, $2017 \quad$ Accepted: December 24, $2017 \quad$ Published: January 02, 2018 \\ Copyright: Kao et al. This is an open-access article distributed under the terms of the Creative Commons Attribution License 3.0 \\ (CC BY 3.0), which permits unrestricted use, distribution, and reproduction in any medium, provided the original author and source \\ are credited.
}

\section{ABSTRACT}

Introductions: Prostate-selective $\alpha$ antagonists are recommended for relief of lower urinary tract symptoms in prostate cancer patients despite uncertainty of fracture risk as an addition to androgen deprivation therapy (ADT). The purpose of this study is to estimate fracture risk associated with these medications in prostate cancer patients who did and did not receive ADT.

Methods: The Taiwan National Health Insurance database was used to identify prostate cancer patients. We identified all 90-day person-quarters exposed to and not exposed to prostate-selective $\alpha$ antagonists. A generalized estimating equation model was used to estimated adjusted odd ratios (ORs) and $95 \%$ confidence intervals (CIs) for fracture associated with prostate-selective $\alpha$ antagonists with consideration for confounding by indication bias using propensity score.

Results: During 1997-2008, 16,601 persons received a diagnosis of prostate cancer, among whom 13,694 received ADT. Among prostate cancer patients receiving ADT, fracture was significantly more common in person-quarters with prostateselective $\alpha$ antagonist use than in quarters without such treatment (OR, 1.08; 95\% CI, 1.00-1.18). Prostate-selective $\alpha$ antagonist use was most strongly associated with femur fracture (OR, 1.22; 95\% CI, 1.09-1.38), followed by skull fracture (OR, 1.29; 95\% CIs: 0.93-1.80). Among patients who did not receive ADT, fracture was more common in person-quarters with prostate-selective $\alpha$ antagonist use than in those without medication use (OR, 1.19; 95\% CI, 0.91-1.55). 


\section{Conclusions: Prostate-selective $\alpha$ antagonist is associated with an increased fracture risk, particular for fractures in skull and femur. Patients should be well- informed on this potential risk before taking prostate-selective $\alpha$ antagonists.}

\section{INTRODUCTION}

Prostate cancer is the fifth most common male cancer in Taiwan [1]. Current guidelines recommend androgen deprivation therapy (ADT) as first-line neoadjuvant and adjuvant therapy in conjunction with radiotherapy for locally advanced prostate cancer and as the standard treatment for disseminated prostate cancer [24]. Despite these recommendations, the balance between the therapeutic benefits and adverse effects of ADT - such as insulin resistance, diabetes mellitus and increased risks of cardiovascular diseases, accelerated bone loss - has not been adequately studied [5-11].

Patients with prostate cancer frequently have urinary symptoms which can adversely affect quality of life. Such symptoms can be relieved by $\alpha$ antagonists. Prostateselective $\alpha$ antagonists such as tamsulosin, silodosin $\left(\alpha_{1 \mathrm{~A}}\right.$ antagonists), and alfuzosin $\left(\alpha_{1}\right.$ antagonists with uroselectivity) are believed to have a better safety profile than nonselective agents because they are less likely to result in side effects such as hypotension, syncope, and dizziness, which may predispose patients with prostate cancer - who are already at risk for osteoporosis because of androgen deprivation - to falls and fracture [12-16].

Results of studies on the safety of prostate-selective $\alpha$ antagonists for prostate cancer patients receiving androgen deprivation have been contradictory, particularly those related to the risks of falls and fracture [17-19]. In addition, there is limited evidence regarding fracture risk associated with prostate-selective $\alpha$ antagonists, with or without a history of ADT. Therefore, we estimated the effects of prostate-selective $\alpha$ antagonists on fracture risk among prostate cancer patients receiving ADT or not receiving ADT in Taiwan between 1997 and 2008.

\section{RESULTS}

\section{Patient characteristics}

During 1997-2008, a total of 16,601 patients were eligible for this study. Among them, 13,694 of received ADT. Among patients receiving ADT, 9,686 (70.7\%) used one or more types of prostate-selective $\alpha$ antagonist and $4,008(29.3 \%)$ never used a prostate-selective $\alpha$ antagonist. Among patients without a history of ADT ( $\mathrm{n}=2907), 1668$ had been prescribed prostate-selective $\alpha$ antagonists (Figure 1). The characteristics of the patients at diagnosis are shown in Table 1. The absolute standardized mean differences of the patients' characteristics after propensity score weighting are listed in Supplementary Tables 9 and 10.

\section{Fracture risk in patients with prostate cancer and in patients with ADT}

Standardized fracture risk was higher among prostate cancer patients than among persons without cancer, with a SIR (95\% CI) of 1.39 (1.27-1.52) (Supplementary Table 4). Among prostate cancer patients, fracture risk of patients with a history of ADT was higher than those without ADT with a HR $(95 \% \mathrm{CI})$ of 1.41 (1.26-1.57) (Supplementary Table 5).

\section{Fracture risk associated with prostate-selective $\alpha$ antagonist use}

Overall there were 563,975 person-quarters for all prostate cancer patients. In patients with a history of ADT, fracture events occurred in 4,012 person-quarters $(0.87 \%)$ of 462,263 person-quarters. Fractures were significantly more frequent in person-quarters with prostate-selective $\alpha$ antagonist use $(1,125 / 103,119$ person-quarters) than in those without such use $(2,887 / 359,144$ person-quarters) among prostate cancer patients with a history of ADT. Prostate-selective $\alpha$ antagonist use was associated with a crude OR for fracture of 1.28 (95\% CI, 1.19-1.39) and an adjusted OR of 1.09 (95\% CI, 1.00-1.18). The three most common fracture sites were the femur $(\mathrm{n}=1,796)$; tibia, fibula, and foot $(\mathrm{n}=950)$; and radius and ulna $(\mathrm{n}=644)$. Among fracture sites, prostate-selective $\alpha$ antagonist use was most strongly associated with femur fracture (crude OR, $1.41 ; 95 \%$ CI, 1.27-1.57; adjusted OR, 1.22; 95\% CI, 1.09-1.38), followed by skull fracture with borderline significance (Table 2).

In the analysis of prostate cancer patients without a history of ADT, 552 person-quarters with fracture were identified among 101,712 person-quarters. There were more fractures in person-quarters with prostateselective $\alpha$ antagonist use (105/12,716 person-quarters) than in quarters without prostate-selective $\alpha$ antagonist use (447/88,996 person-quarters) (crude OR, $1.43 ; 95 \%$ CI, 1.13-1.80; adjusted OR, 1.19; 95\% CI, 0.91-1.55). The most frequent fracture sites were the femur $(n=225)$; tibia, fibula, and foot $(\mathrm{n}=181)$; and radius and ulna $(\mathrm{n}=89)$, and only tibia, fibula, and foot fracture risk significantly increased with prostate-selective $\alpha$ antagonist use (crude OR, 1.63; 95\% CI, 1.09-2.44; adjusted OR, 1.54; 95\% CI, 1.00-2.36). Prostate-selective $\alpha$ antagonist use in patients without a history of ADT was borderline associated with femur fracture (crude OR, 1.51; 95\% CI, 1.07-2.11; adjusted OR, 1.42; 95\% CI, 0.98-2.06) (Table 3). 


\section{Additional analyses}

We expanded the case definition of fracture to outpatient, inpatient and emergency procedures for fracture and repeated the analysis. As shown in Supplementary Tables 6 and 7, prostate-selective $\alpha$ antagonist use was associated with an adjusted OR of 1.05 (95\% CI, 0.97-1.13) for any fracture among patients with ADT use. In patients without a history of ADT, prostate-selective $\alpha$ antagonist use was associated with an adjusted OR of 1.10 (95\% CI, 0.86-1.41). For femur fracture, OR was 1.2 (95\% CI, 1.07-1.35) among patients with ADT use. For patients without ADT use, the adjusted OR $(95 \% \mathrm{CI})$ for femur fracture was $1.50(1.05-2.13)$. In Supplementary Table 8, for patients with ADT use history who are older than or equal to 73 years old, adjusted OR of any fracture is $1.09(95 \% \mathrm{CI}, 1.00-1.20)$ and OR is 1.00 ( $95 \%$ CI, 0.80-1.25) for those younger than 73 years old. Adjusted OR are 0.8 (95\% CI 0.42-1.54, age $<73$ year-old) and 1.26 (95\% CI $0.96-1.69, \geq 73$ year-old) for patients without ADT history.

\section{DISCUSSION}

\section{Principal findings}

The Prostate Cancer Survivorship Care Guidelines of the American Society of Clinical Oncology recommend that physicians consider $\alpha$-blockers for slow stream urinary dysfunction [20]. However, the safety of prostateselective $\alpha$ antagonists remains a matter of debate. In this study, use of prostate-selective $\alpha$ antagonists was associated with a 1.08-fold increase in fracture risk among prostate cancer patients with a history of ADT. Analysis of prostate cancer patients without a history of ADT use revealed a similar increase in fracture risk (OR, 1.19). In addition, prostate-selective $\alpha$ antagonists were associated with increased risks of skull (OR, 1.29) and femur fracture (OR, 1.22), which suggests that postural hypotension associated with these drugs was the main predisposing factor. Our findings indicate that fracture risk is higher among prostate-selective $\alpha$ antagonist users than among

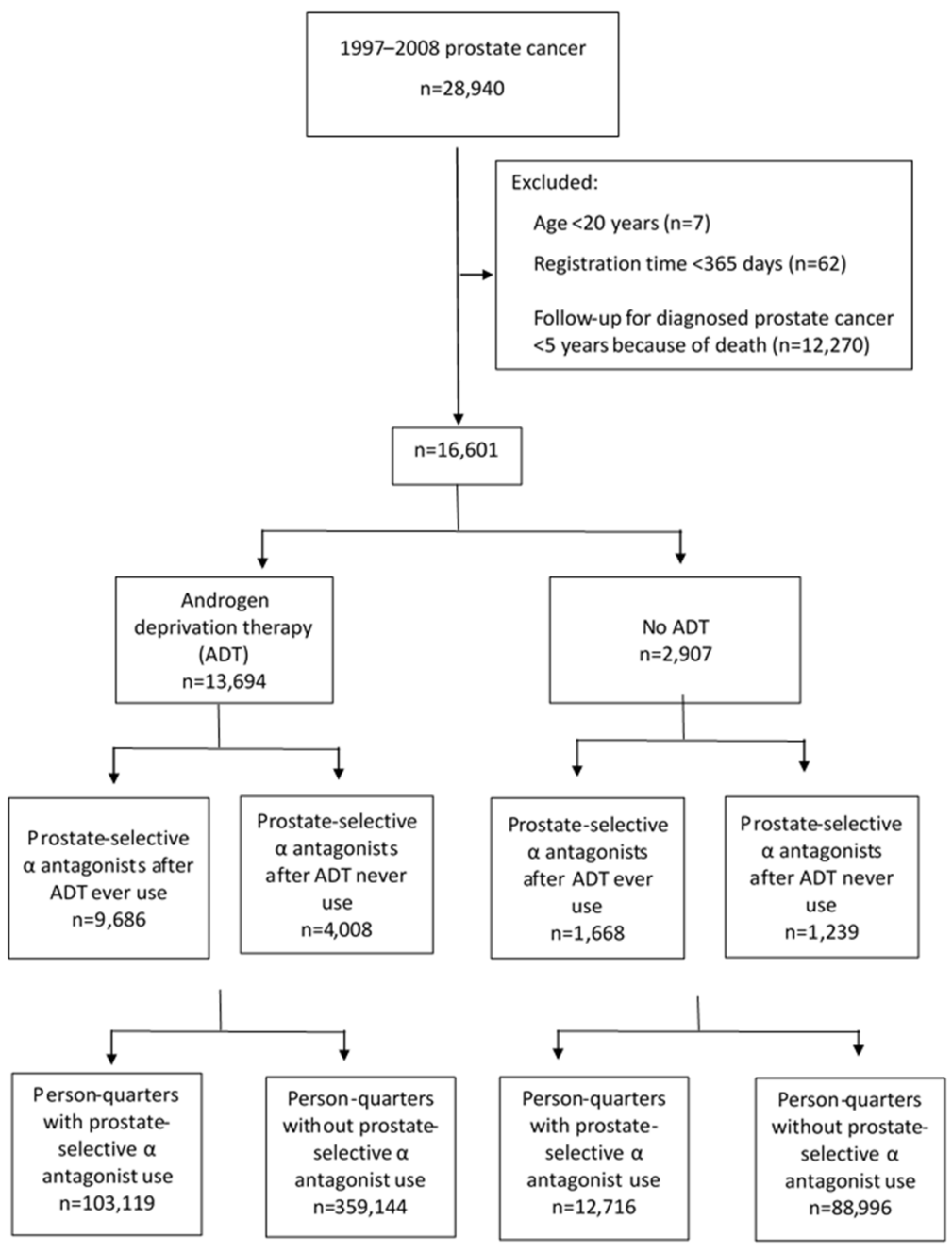

Figure 1: Flow of included patients for analyses with numbers of excluded observations. 
Table 1: Characteristics of study population

\begin{tabular}{|c|c|c|c|c|c|c|c|c|c|c|}
\hline \multirow[b]{2}{*}{ Characteristics } & \multicolumn{5}{|c|}{ With androgen deprivation therapy } & \multicolumn{5}{|c|}{ Without androgen deprivation therapy } \\
\hline & \multicolumn{2}{|c|}{$\begin{array}{l}\text { Any prostate- } \\
\text { selective } \alpha \\
\text { antagonist use } \\
(n=9,686)\end{array}$} & \multicolumn{2}{|c|}{$\begin{array}{l}\text { No prostate- } \\
\text { selective } \alpha \\
\text { antagonist use } \\
(\mathrm{n}=\mathbf{4 , 0 0 8 )}\end{array}$} & p value & \multicolumn{2}{|c|}{$\begin{array}{l}\text { Any prostate- } \\
\text { selective } \alpha \\
\text { antagonist use } \\
(n=1,668)\end{array}$} & \multicolumn{2}{|c|}{$\begin{array}{c}\text { No prostate- } \\
\text { selective } \alpha \\
\text { antagonist use } \\
(n=1,239)\end{array}$} & p value \\
\hline $\begin{array}{l}\text { Age (years) (mean } \pm \\
\text { standard deviation) }\end{array}$ & \multicolumn{2}{|c|}{$73.02 \pm 7.27$} & \multicolumn{2}{|c|}{$70.73 \pm 8.17$} & $<0.0001$ & \multicolumn{2}{|c|}{$70.52 \pm 7.69$} & \multicolumn{2}{|c|}{$67.62 \pm 9.10$} & $<0.0001$ \\
\hline $\begin{array}{l}\text { Charlson Comorbidity } \\
\text { Index }\end{array}$ & \multicolumn{2}{|c|}{$3.85 \pm 2.14$} & \multicolumn{2}{|c|}{$3.57 \pm 2.15$} & $<0.0001$ & \multicolumn{2}{|c|}{$3.50 \pm 1.65$} & \multicolumn{2}{|c|}{$3.11 \pm 1.59$} & $<0.0001$ \\
\hline$\leq 3$ & 5,561 & $(57.41)$ & 2,668 & $(66.57)$ & $<0.0001$ & 1037 & $(62.17)$ & 916 & $(54.92)$ & $<0.0001$ \\
\hline$>3$ & 4,125 & $(42.59)$ & 1,340 & $(33.43)$ & & 631 & $(37.83)$ & 323 & (19.36) & \\
\hline \multicolumn{11}{|l|}{ Comorbidities } \\
\hline Hypertension $^{\dagger}$ & 5,318 & $(54.90)$ & 1,893 & $(47.23)$ & $<0.0001$ & 909 & $(54.50)$ & 556 & $(33.33)$ & $<0.0001$ \\
\hline Osteoporosis & 911 & $(9.41)$ & 271 & $(6.76)$ & $<0.0001$ & 170 & $(10.19)$ & 79 & $(4.74)$ & 0.0003 \\
\hline \multicolumn{11}{|l|}{ Medication use, No. $(\%)^{\dagger}$} \\
\hline Calcium channel blockers & 5,229 & (53.99) & 1,853 & $(46.23)$ & $<0.0001$ & 866 & $(51.92)$ & 511 & (30.64) & $<0.0001$ \\
\hline $\mathrm{ACE}$ inhibitors or $\mathrm{ARB}$ & 3,799 & $(39.22)$ & 1,343 & $(33.51)$ & $<0.0001$ & 625 & $(37.47)$ & 383 & $(22.96)$ & 0.0002 \\
\hline$\beta$ blockers & 3,687 & $(38.07)$ & 1,343 & $(33.51)$ & $<0.0001$ & 679 & $(40.71)$ & 387 & $(23.20)$ & $<0.0001$ \\
\hline$\alpha$ blockers ${ }^{\S}$ & 7,373 & $(76.12)$ & 2,855 & $(71.23)$ & $<0.0001$ & 1189 & $(71.28)$ & 763 & $(45.74)$ & $<0.0001$ \\
\hline Hydrazinophthalazine & 784 & (8.09) & 327 & $(8.16)$ & 0.8999 & 126 & $(7.55)$ & 61 & $(3.66)$ & 0.0043 \\
\hline $\mathrm{K}+$ sparing diuretics & 1,002 & $(10.34)$ & 313 & $(7.81)$ & $<0.0001$ & 129 & $(7.73)$ & 79 & $(4.74)$ & 0.1602 \\
\hline Loop diuretics & 3,129 & $(32.30)$ & 1,196 & $(29.84)$ & 0.0048 & 522 & $(31.29)$ & 371 & $(22.24)$ & 0.4347 \\
\hline Thiazide diuretics & 2,596 & $(26.80)$ & 893 & $(22.28)$ & $<0.0001$ & 423 & $(25.36)$ & 228 & $(13.67)$ & $<0.0001$ \\
\hline Benzodiazepines & 7,304 & $(75.41)$ & 2,705 & $(67.49)$ & $<0.0001$ & 1252 & $(75.06)$ & 762 & $(45.68)$ & $<0.0001$ \\
\hline Bisphosphonates & 83 & $(0.86)$ & 16 & $(0.40)$ & 0.0040 & 7 & $(0.42)$ & 4 & $(0.24)$ & 0.6741 \\
\hline Glucocorticoids & 5316 & $(54.88)$ & 1983 & $(49.48)$ & $<0.0001$ & 905 & $(54.26)$ & 579 & $(34.71)$ & $<0.0001$ \\
\hline Narcotics & 2,655 & $(27.41)$ & 1,082 & $(27.00)$ & 0.6201 & 495 & $(29.68)$ & 341 & $(20.44)$ & 0.2045 \\
\hline $\begin{array}{l}\text { Overactive-bladder } \\
\text { medications }\end{array}$ & 3,025 & $(31.23)$ & 1,081 & $(26.97)$ & $<0.0001$ & 516 & (30.94) & 306 & $(18.35)$ & 0.0002 \\
\hline Proton pump inhibitors & 1,386 & $(14.31)$ & 458 & $(11.43)$ & $<0.0001$ & 268 & $(16.07)$ & 160 & $(9.59)$ & 0.0176 \\
\hline Statins & 1,312 & $(13.55)$ & 495 & $(12.35)$ & 0.0601 & 280 & $(16.79)$ & 163 & $(9.77)$ & 0.0071 \\
\hline 5 - $\alpha$-reductase inhibitors & 2,603 & $(26.87)$ & 895 & $(22.33)$ & $<0.0001$ & 117 & $(7.01)$ & 60 & $(3.60)$ & 0.0155 \\
\hline NSAIDs & 8,995 & $(92.87)$ & 3,561 & $(88.85)$ & $<0.0001$ & 1550 & $(92.93)$ & 1,076 & $(64.51)$ & $<0.0001$ \\
\hline Insulin & 502 & $(5.18)$ & 183 & $(4.57)$ & 0.1319 & 80 & $(4.80)$ & 58 & $(3.48)$ & 0.8854 \\
\hline Anticoagulants & 693 & $(7.15)$ & 245 & $(6.11)$ & 0.0281 & 150 & $(8.99)$ & 117 & $(7.01)$ & 0.6776 \\
\hline Anticonvulsants & 1,307 & $(13.49)$ & 332 & $(8.28)$ & $<0.0001$ & 237 & $(14.21)$ & 112 & $(6.71)$ & $<0.0001$ \\
\hline Lipid lowering agents & 1,790 & $(18.48)$ & 686 & $(17.12)$ & 0.0591 & 353 & $(21.16)$ & 217 & $(13.01)$ & 0.0143 \\
\hline \multicolumn{11}{|l|}{ Treatment } \\
\hline Radiotherapy & 4,730 & $(48.83)$ & 1,310 & $(32.68)$ & $<0.0001$ & 526 & (31.53) & 190 & (11.39) & $<0.0001$ \\
\hline Radical prostatectomy & 1,070 & $(11.05)$ & 1,244 & $(31.04)$ & $<0.0001$ & 694 & (41.61) & 720 & (43.17) & $<0.0001$ \\
\hline
\end{tabular}

(Continued) 


\begin{tabular}{|c|c|c|c|c|c|c|c|c|c|c|}
\hline \multirow{2}{*}{$\begin{array}{l}\text { Characteristics } \\
\text { Place of residence, No. (\%) }\end{array}$} & \multicolumn{5}{|c|}{ With androgen deprivation therapy } & \multicolumn{5}{|c|}{ Without androgen deprivation therapy } \\
\hline & \multicolumn{2}{|c|}{$\begin{array}{l}\text { Any prostate- } \\
\text { selective } \alpha \\
\text { antagonist use } \\
(\mathbf{n}=\mathbf{9 , 6 8 6 )}\end{array}$} & \multicolumn{2}{|c|}{$\begin{array}{l}\text { No prostate- } \\
\text { selective } \alpha \\
\text { antagonist use } \\
(\mathrm{n}=\mathbf{4 , 0 0 8 )}\end{array}$} & $\begin{array}{l}\text { p value } \\
<0.0001\end{array}$ & \multicolumn{2}{|c|}{$\begin{array}{l}\text { Any prostate- } \\
\text { selective } \alpha \\
\text { antagonist use } \\
(\mathbf{n}=\mathbf{1 , 6 6 8 )}\end{array}$} & \multicolumn{2}{|c|}{$\begin{array}{l}\text { No prostate- } \\
\text { selective } \alpha \\
\text { antagonist use } \\
\quad(n=1,239)\end{array}$} & $\begin{array}{l}\text { p value } \\
0.3899\end{array}$ \\
\hline Urban & 2,843 & $(29.35)$ & 1,366 & $(34.08)$ & & 547 & $(32.79)$ & 428 & $(25.66)$ & \\
\hline Suburban & 2,640 & $(27.26)$ & 1,030 & $(25.70)$ & & 471 & $(28.24)$ & 329 & $(19.72)$ & \\
\hline Rural & 4,002 & $(41.32)$ & 1519 & $(37.90)$ & & 616 & $(36.93)$ & 448 & $(26.86)$ & \\
\hline Unknown & 201 & $(2.08)$ & 93 & $(2.32)$ & & 34 & $(2.04)$ & 34 & $(2.04)$ & \\
\hline Income level, No. (\%) & & & & & $<0.0001$ & & & & & $<0.0001$ \\
\hline Quintile 1 & 2460 & $(25.40)$ & 896 & $(22.36)$ & & 467 & $(28.00)$ & 285 & $(23.00)$ & \\
\hline Quintile 2 & 1376 & $(14.21)$ & 523 & $(13.05)$ & & 240 & (14.39) & 168 & $(13.56)$ & \\
\hline Quintile 3 & 2251 & $(23.24)$ & 874 & $(21.81)$ & & 318 & (19.06) & 228 & $(18.40)$ & \\
\hline Quintile 4 & 1797 & $(18.55)$ & 731 & $(18.24)$ & & 364 & $(21.82)$ & 242 & (19.53) & \\
\hline Quintile 5 & 1765 & $(18.22)$ & 965 & $(24.08)$ & & 278 & $(16.67)$ & 302 & $(24.37)$ & \\
\hline Unknown & 37 & $(0.38)$ & 19 & $(0.47)$ & & 1 & $(0.06)$ & 14 & $(1.13)$ & \\
\hline Occupation, No. (\%) & & & & & $<0.0001$ & & & & & $<0.0001$ \\
\hline $\begin{array}{l}\text { Dependent of insured } \\
\text { individual }\end{array}$ & 2,479 & $(25.59)$ & 1,023 & $(25.52)$ & & 401 & $(24.04)$ & 271 & $(16.25)$ & \\
\hline $\begin{array}{l}\text { Civil servant, teacher, } \\
\text { military personnel, and } \\
\text { veteran }\end{array}$ & 1,085 & $(11.20)$ & 435 & $(10.85)$ & & 212 & $(12.71)$ & 149 & $(8.93)$ & \\
\hline $\begin{array}{l}\text { Non-manual workers and } \\
\text { professionals }\end{array}$ & 746 & $(7.70)$ & 497 & $(12.40)$ & & 163 & $(9.77)$ & 198 & (11.87) & \\
\hline Manual workers & 3,144 & $(32.46)$ & 1,186 & $(29.59)$ & & 446 & $(26.74)$ & 327 & $(19.60)$ & \\
\hline Other & 2,232 & $(23.04)$ & 867 & $(21.63)$ & & 446 & $(26.74)$ & 294 & $(17.63)$ & \\
\hline
\end{tabular}

${ }^{\dagger}$ Diagnosed during the 3 years before prostate cancer diagnosis.

* Diagnosed any time before prostate cancer diagnosis.

$\S$ Tamsulosin, silodosin, alfuzosin were excluded.

"At any time.

nonusers, irrespective of ADT use, presumably because of the adverse effects of postural hypotension.

A previous study reported a 2.8-fold increase in the risk of fracture requiring hospitalization among prostate cancer patients receiving ADT [10]. Among patients who received GnRH agonists, fracture risk was 38\% higher than for patients who did not receive GnRH agonists and $103 \%$ higher than for patients who underwent orchiectomy [11]. In the present study, fracture incidence was higher among patients who received ADT than among those who did not (adjusted HR, 1.41) in Supplementary Table 5. In fracture site analysis, only the risk of femoral fracture was significantly increased, regardless of ADT exposure. The risk of skull fracture was borderline-significantly higher only in patients with a history of ADT. However, there was small difference in the magnitude of relative risks associated with prostate-selective $\alpha$ antagonists in patients who received ADT (OR: 1.08) and those who did not (OR: 1.19). This might be due to higher fracture percentage in patients who received ADT without prostate-selective $\alpha$ antagonists use (ADT vs. no $\mathrm{ADT}=0.8 \%$ vs. $0.5 \%$ ). Although the difference in relative risk was modest, the absolute increase in events in personal-quarters for patients who received ADT was much higher than for those who did not receive ADT, because patients receiving ADT had more risk factors for fracture at baseline [10, 11]. This could result in more fracture-related deaths among these patients [10]. 
Table 2: Fracture risk in prostate cancer patients with a history of androgen deprivation therapy with and without prostate-selective $\alpha$ antagonist use based on fracture diagnosed in emergency and inpatient departments using propensity score weighting method

\begin{tabular}{|c|c|c|c|c|c|c|c|c|c|}
\hline \multirow{2}{*}{$\begin{array}{l}\text { Fracture sites } \\
\text { All fractures }\end{array}$} & \multirow{2}{*}{$\begin{array}{c}\begin{array}{c}\text { Full sample } \\
(\mathbf{n}=\mathbf{4 6 2 , 2 6 3 )}\end{array} \\
4,012\end{array}$} & \multicolumn{2}{|c|}{$\begin{array}{l}\text { Person-quarters } \\
\text { with prostate- } \\
\text { selective } \alpha \\
\text { antagonist use } \\
(\mathrm{n}=\mathbf{1 0 3 , 1 1 9 )}\end{array}$} & \multicolumn{2}{|c|}{$\begin{array}{l}\text { Person-quarters } \\
\text { without prostate- } \\
\text { selective } \alpha \\
\text { antagonist use } \\
(\mathrm{n}=\mathbf{3 5 9 , 1 4 4 )}\end{array}$} & \multicolumn{2}{|c|}{ Crude OR $^{\mathrm{a}}(95 \% \mathrm{CI})$} & \multicolumn{2}{|c|}{$\begin{array}{l}\text { Adjusted OR } \\
\text { (95\% CI) }\end{array}$} \\
\hline & & 1,125 & $(1 \cdot 09)$ & 2,887 & $(0 \cdot 80)$ & 1.28 & $(1.19-1.39)^{*}$ & 1.08 & $(1.00-1.18)^{*}$ \\
\hline Skull & 194 & 60 & $(0 \cdot 06)$ & 134 & $(0 \cdot 04)$ & 1.49 & $(1.10-2.03)^{*}$ & 1.29 & $(0.93-1.80)$ \\
\hline Vertebrae & 585 & 179 & $(0 \cdot 17)$ & 406 & $(0 \cdot 11)$ & 1.41 & $(1.16-1.71)^{*}$ & 1.09 & $(0.88-1.35)$ \\
\hline Rib & 330 & 88 & $(0 \cdot 09)$ & 242 & $(0 \cdot 07)$ & 1.22 & $(0.94-1.57)$ & 1.00 & $(0.76-1.32)$ \\
\hline Pelvis & 64 & 17 & $(0 \cdot 02)$ & 47 & $(0 \cdot 01)$ & 1.14 & $(0.64-2.01)$ & 0.98 & $(0.54-1.78)$ \\
\hline Clavicle & 161 & 30 & $(0 \cdot 03)$ & 131 & $(0 \cdot 04)$ & 0.82 & $(0.55-1.22)$ & 0.66 & $(0.44-1.01)$ \\
\hline Scapula & 35 & 7 & $(0 \cdot 01)$ & 28 & $(0 \cdot 01)$ & 0.89 & $(0.39-2.06)$ & 0.77 & $(0.32-1.84)$ \\
\hline Humerus & 634 & 150 & $(0 \cdot 15)$ & 484 & $(0 \cdot 13)$ & 1.04 & $(0.86-1.26)$ & 0.88 & $(0.72-1.08)$ \\
\hline Radius and ulna & 644 & 148 & $(0 \cdot 14)$ & 496 & $(0 \cdot 14)$ & 1.00 & $(0.82-1.22)$ & 0.82 & $(0.67-1.01)$ \\
\hline Hand & 340 & 89 & $(0 \cdot 09)$ & 251 & $(0 \cdot 07)$ & 1.23 & $(0.96-1.58)$ & 1.16 & $(0.89-1.52)$ \\
\hline Femur & 1,796 & 548 & $(0 \cdot 53)$ & 1248 & $(0 \cdot 35)$ & 1.41 & $(1.27-1.57)^{*}$ & 1.22 & $(1.09-1.38)^{*}$ \\
\hline Tibia, fibula, and foot & 950 & 254 & $(0 \cdot 25)$ & 696 & $(0 \cdot 20)$ & 1.27 & $(1.09-1.47)^{*}$ & 1.12 & $(0.95-1.31)$ \\
\hline
\end{tabular}

${ }^{a}$ Adjusted for age.

${ }^{\mathrm{b}}$ Using inverse probability of treatment weights of propensity scores ( medical utilization, Charlson Comorbidity Index, hypertension, osteoporosis, calcium channel blocker, ACE inhibitor, ARB, diuretics-K+ sparing, diuretics-loop diuretics, diuretics-thiazide, $\beta$ blockers, $\alpha$ blockers (tamsulosin, silodosin, alfuzosin were excluded), benzodiazepine, bisphosphonates, glucocorticoids, narcotics, overactive bladder medication, proton pump inhibitors, statin, 5- $\alpha$-reductase inhibitors, hydrazinophthalazine, NSAID, insulin, anticoagulants, anticonvulsants, lipid lowering agents, echocardiography (EKG), bone mineral density test, bone scan, cardiac stress test, CT head, radiotherapy, radical prostectomy, place of residence, income levels, occupation). ${ }^{*} \mathrm{p}<0.05$.

\section{Comparison with previous findings}

Two large population-based studies reported that use of nonselective $\alpha$ antagonists was associated with increased risk of hypotension-related adverse events and hip/femur fracture within 4 months after the start of therapy [12]. These adverse effects were more common during the initial stage after starting a new treatment (for both prostate-selective and nonselective antagonists) [21]. However fracture risk was not associated with use of modified-release doxazosin in The Health Improvement Network (THIN) UK database case-control study, [22] and a Danish nationwide case-control study reported no association between fracture risk and use of $\alpha$ antagonists (for both prostate-selective and nonselective antagonists) [23]. A recent population-based retrospective cohort study reported a temporal association between tamsulosin use and severe hypotension during the first 8 weeks after initiating treatment and 8 weeks after restarting treatment,
[17] and a matched cohort study found significantly higher risks of falling and fracture in patients treated with alfuzosin, tamsulosin, or silodosin [18]. However, change in blood pressure on standing did not significantly differ between tamsulosin and placebo in a relatively small double-blind phase III study, [24] and rates of dizziness, headache, and hypotension were relatively low in a large cohort questionnaire study [25].

\section{Study strengths and limitations}

This nationwide population-based study included newly diagnosed prostate cancer patients followed-up for over a decade. With propensity score weighting in each person-quarter, we considered complex prescription decision-making for prostate-selective $\alpha$ antagonists, which is based on changes in patient condition. In addition, we conducted multiple sensitivity analyses which all indicate an increased risk for femur fracture in prostate 
Table 3: Fracture risk in prostate cancer patients without a history of androgen deprivation therapy with and without prostate-selective $\alpha$ antagonist use based on fracture diagnosed in emergency and inpatient departments using propensity score weighting method

\begin{tabular}{|c|c|c|c|c|c|c|c|c|c|}
\hline \multirow{2}{*}{$\begin{array}{l}\text { Fracture sites } \\
\text { Overall fracture }\end{array}$} & \multirow{2}{*}{$\begin{array}{c}\begin{array}{c}\text { Full sample } \\
(\mathbf{n}=101,712)\end{array} \\
552\end{array}$} & \multicolumn{2}{|c|}{$\begin{array}{c}\text { Person- } \\
\text { quarters with } \\
\text { prostate- } \\
\text { selective } \alpha \\
\text { antagonist use } \\
(\mathrm{n}=12,716)\end{array}$} & \multicolumn{2}{|c|}{$\begin{array}{l}\text { Person-quarters } \\
\text { without prostate- } \\
\text { selective } \alpha \\
\text { antagonist use } \\
(\mathrm{n}=\mathbf{8 8 , 9 9 6 )}\end{array}$} & \multicolumn{2}{|c|}{ Crude OR ${ }^{\text {a }}(95 \% \mathrm{CI})$} & \multicolumn{2}{|c|}{$\begin{array}{l}\text { Adjusted } \mathrm{OR}^{\mathrm{b}} \\
\quad(95 \% \mathrm{CI})\end{array}$} \\
\hline & & 105 & $(0 \cdot 83)$ & 447 & $(0 \cdot 50)$ & $1 \cdot 43$ & $(1 \cdot 13-1 \cdot 80)^{*}$ & 1.19 & $(0.91-1.55)$ \\
\hline Skull & 32 & 8 & $(0 \cdot 06)$ & 24 & $(0 \cdot 03)$ & $2 \cdot 02$ & $(0 \cdot 91-4 \cdot 49)$ & 1.36 & $(0.45-4.13)$ \\
\hline Vertebrae & 67 & 11 & $(0 \cdot 09)$ & 56 & $(0 \cdot 06)$ & $1 \cdot 06$ & $(0 \cdot 52-2 \cdot 13)$ & 0.76 & $(0.35-1.63)$ \\
\hline Rib & 54 & 9 & $(0 \cdot 07)$ & 45 & $(0 \cdot 05)$ & $1 \cdot 36$ & $(0 \cdot 67-2 \cdot 73)$ & 1.13 & $(0.50-2.55)$ \\
\hline Pelvis & 12 & 2 & $(0 \cdot 02)$ & 10 & $(0 \cdot 01)$ & $1 \cdot 45$ & $(0 \cdot 29-7 \cdot 26)$ & 1.10 & $(0.21-5.88)$ \\
\hline Clavicle & 25 & 4 & $(0 \cdot 03)$ & 21 & $(0 \cdot 02)$ & $1 \cdot 32$ & $(0 \cdot 45-3 \cdot 93)$ & 0.95 & $(0.29-3.09)$ \\
\hline Scapula & 5 & 0 & $(0 \cdot 00)$ & 5 & $(0 \cdot 01)$ & & $\mathrm{N} / \mathrm{A}$ & & $\mathrm{N} / \mathrm{A}$ \\
\hline Humerus & 68 & 11 & $(0 \cdot 09)$ & 57 & $(0 \cdot 06)$ & $1 \cdot 19$ & $(0 \cdot 62-2 \cdot 27)$ & 0.82 & $(0.39-1.72)$ \\
\hline Radius and ulna & 89 & 10 & $(0 \cdot 08)$ & 79 & $(0 \cdot 09)$ & $0 \cdot 82$ & $(0 \cdot 42-1 \cdot 59)$ & 0.75 & $(0.36-1.54)$ \\
\hline Hand & 57 & 11 & $(0 \cdot 09)$ & 46 & $(0 \cdot 05)$ & $1 \cdot 56$ & $(0 \cdot 76-3 \cdot 18)$ & 1.9 & $(0.88-4.10)$ \\
\hline Femur & 225 & 46 & $(0 \cdot 36)$ & 179 & $(0 \cdot 20)$ & $1 \cdot 51$ & $(1 \cdot 07-2 \cdot 11)^{*}$ & 1.42 & $(0.98-2.06)$ \\
\hline Tibia, fibula, and foot & 181 & 37 & $(0 \cdot 29)$ & 144 & $(0 \cdot 16)$ & $1 \cdot 63$ & $(1 \cdot 09-2 \cdot 44)^{*}$ & 1.54 & $(1.00-2.36)^{*}$ \\
\hline
\end{tabular}

${ }^{a}$ Adjusted for age.

${ }^{\mathrm{b}}$ Using inverse probability of treatment weights of propensity scores ( medical utilization, Charlson Comorbidity Index, hypertension, osteoporosis, calcium channel blocker, ACE inhibitor, ARB, diuretics-K+ sparing, diuretics-loop diuretics, diuretics-thiazide, $\beta$ blockers, $\alpha$ blockers (tamsulosin, silodosin, alfuzosin were excluded), benzodiazepine, bisphosphonates, glucocorticoids, narcotics, overactive bladder medication, proton pump inhibitors, statin, 5- $\alpha$-reductase inhibitors, hydrazinophthalazine, NSAID, insulin, anticoagulants, anticonvulsants, lipid lowering agents, echocardiography (EKG), bone mineral density test, bone scan, cardiac stress test, CT head, radiotherapy, radical prostectomy, place of residence, income levels, occupation). ${ }^{*} \mathrm{p}<0.05$.

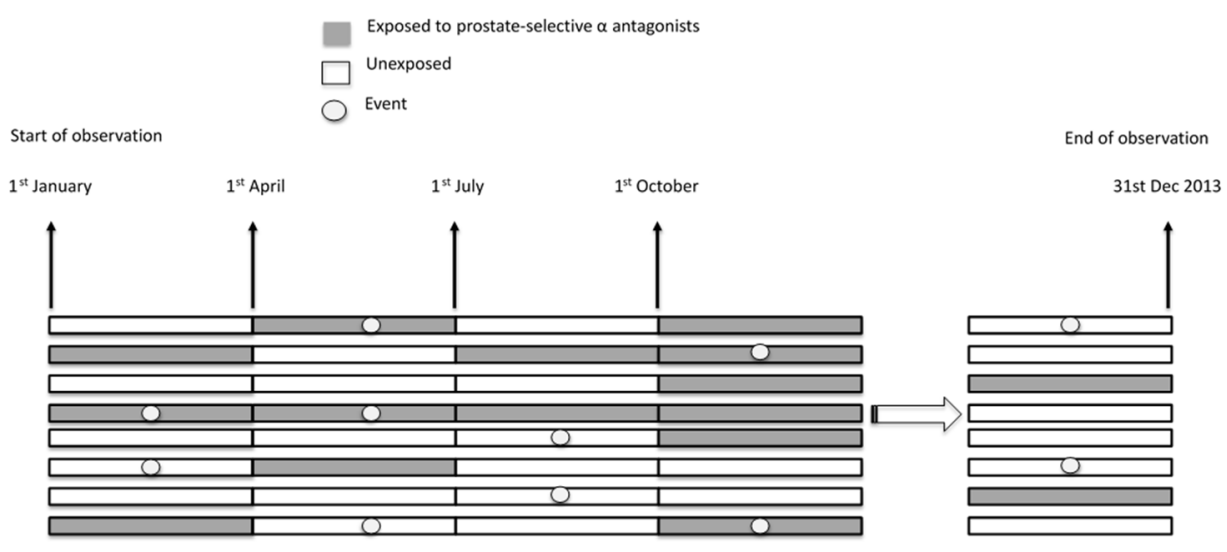

Figure 2: Schematic presentation of person-quarter design. 
cancer patients using prostate-selective $\alpha$ antagonist with or without ADT. Therefore, although the increase in fracture risk associated with prostate-selective $\alpha$ antagonist was modest, it is nevertheless a genuine risk, particularly for femur fracture that should be considered by physicians.

This study has several limitations. Firstly, it is possible that some fractures were related to disease progression in patients with bony metastases. However, in our study, we only included the patients with minimum survival time of 5 years to reduce the impact of the pathologic fracture and based on the Denmark cohort study, the 5-year-survival probability of the prostate cancer patients with whenever bony metastases was 2.7\% (95\% CI 2.2-3.4) [26]. Secondly, our data of radiotherapy course had no details of prostate irradiation only or prostate plus pelvic irradiation. Nevertheless the cumulative incidence of insufficiency fracture in pelvic irradiation was relative low with $6.8 \%$ [27]. Although we didn't adjust the irradiation specific to pelvic region, we still adjusted the factor of receiving radiotherapy or not for the fracture result. Third, we didn't have daily blood pressure with direct connection with our theory of drug-induced hypotension and fracture. In another way, we try to use these patients' hypertensive medications to eliminate the impact. Fourth, some potential confounding factors may not have been measured in our study, such as cancer stage, tumor grade, relatively rare comorbidities and limited mobility [13]. However, these factors are unlikely to change the increase in fracture risk associated with prostate-selective $\alpha$ antagonist use in prostate cancer patients [18].

\section{MATERIALS AND METHODS}

\section{Design and setting}

This study was approved by the institutional review board of Chang Gung Memorial Hospital in Taiwan (approval number 104-7041B). The primary data source was the Taiwan National Health Insurance (NHI) database. The NHI system was established in 1995 and is a singlepayer insurance. Approximately 23 million beneficiaries were registered in 2013 with a coverage rate of $99.5 \%$ [28]. The accuracy of the recorded diagnoses is high, and the validity of the database has been confirmed in multiple studies [29-33]. The database is anonymized before its release for research use.

In Taiwan, patients with major diseases, including solid and haematological malignancies, receive a waiver of medical co-payment (catastrophic illness certificate). The unique personal identifier, diagnosis, demographic data, application date, and other data for patients receiving a medical waiver are recorded in the Registry of Patients with Catastrophic Illnesses, which we used to identify patients with prostate cancer in the present study
(International Classification of Disease, ninth revision, clinical modification [ICD-9-CM] code, 185.0).

To ascertain the validity of prostate cancer diagnoses in the NHI database, we linked the NHI with the National Cancer Registry, which served as the reference standard. We identified 40,303 incident cases of prostate cancer between 2001 and 2012, as indicated by a catastrophic illness certificate for prostate cancer. Agreement between the NHI database and National Cancer Registry was good, with a sensitivity of 0.92 , a specificity of 1 , a positive predictive value of 0.93 , and a negative predictive value of 1 [34].

\section{Patient population and covariates}

We established a cohort of patients aged 20 years or older who received a new diagnosis of prostate cancer between 1 January 1997 and 31 December 2008 (Figure 1). A minimum of one year of observation before the date of diagnosis and five years of follow-up were required for inclusion in the analysis [26]. Patients were grouped according to use of ADT (including gonadotropinreleasing hormone agonists $(\mathrm{GnRH})$, anti-androgen agents, and bilateral orchiectomy). Each calendar year was partitioned into four quarters for each patient and each year after ADT (Figure 2). The analytic unit was thus one quarter. We identified all person-quarters with exposures to tamsulosin, alfuzosin, or silodosin and those without such exposures.

The patients' demographics, comorbidities, medication use, treatment of prostate cancer, and health care use were analysed as covariates [18]. Patient demographics included age, gender, place of residence, income level, and occupation. The Charlson comorbidity index, [35] was used to evaluate patient comorbidity burden in relation to 17 diagnostic conditions three years before the cancer diagnosis. Other comorbidities, including hypertension three years before cancer diagnosis and osteoporosis at any time before diagnosis, were also evaluated. Twenty-two types of medications were assessed [18].

\section{Study outcomes}

Our primary outcome was any or site-specific fracture. The unit of analysis was a person-quarter. We used ICD-9-CM codes to identify fractures recorded in emergency and inpatient departments and procedure codes for fracture management in inpatient, and emergency departments and confirmed with ICD-9-CM codes. Fractures were classified according to sites. Fractures were evaluated in each person-quarter and therefore one patient can have multiple events. In addition, we performed a sensitivity analysis using the aforementioned procedure codes for fracture management not only in emergency and inpatient departments, but also the codes in outpatient clinics, and then classified fracture location. The full code 
lists for ADT, outcome definition and covariates are shown in the Supplementary Tables 1, 2 and 3.

\section{Statistical analysis}

In our primary analyses, we used multivariable logistic regression to model the relation between fracture and use of prostate-selective $\alpha$ antagonists among patients with prostate cancer, with or without ADT. The prescription of prostate-selective $\alpha$ antagonist may subject to the "confounding by indication" as a result of nonrandom treatment allocation [36] which was accounted for by utilising propensity score weighting methods [37]. The propensity score is the predicted probability that a patient is prescribed prostate-selective $\alpha$ antagonist during a quarter using logistic regression to model this group as a function of potential confounders of the grouping status and study outcomes [37, 38]. For each personquarter, an inverse probability of treatment weights of specific propensity score was calculated using logistic regression with covariates pertinent to the first date of the quarter, as determined by analysis of the previous quarter and balance covariates (medical utilization, Charlson Comorbidity Index, hypertension, osteoporosis, calcium channel blocker, ACE inhibitor, ARB, diuretics-K+ sparing, diuretics-loop diuretics, diuretics-thiazide, beta blocker, alpha blocker, benzodiazepine, bisphosphonates, glucocorticoids, narcotics, overactive bladder medication, proton pump inhibitors, statin, 5 - $\alpha$-reductase inhibitors, hydrazinophthalazine, NSAID, insulin, anticoagulants, anticonvulsants, lipid lowering agents, echocardiography (EKG), bone mineral density test, bone scan, cardiac stress test, CT head, radiotherapy, radical prostatectomy, place of residence, income levels, occupation) across the two study groups. To account for intra-individual correlation in outcomes across quarters, we used multivariable logistic regression with a generalized estimating equation model to calculate crude odd ratios (ORs), $95 \%$ CIs, and adjusted estimates. The correlation structure was based on autoregressive model.

We further performed additional analysis. Firstly, we compared the overall and site-specific fracture incidence between patients with prostate cancer with the general population. Standardized incidence ratio (SIR) for fracture was calculated. The SIR is the ratio of the actual fracture number of patients with cancer in this study to the expected fracture number of patients with cancer based on age- and time-specific incidence rates in 5-year age intervals in the general population (Supplementary Table 4). To compare the risk of fracture between people with or without ADT, we further used Cox proportional hazards model to estimate hazard ratio (HR) for fracture, adjusting for the aforementioned covariates. ADT use was time dependent variable (Supplementary Table 5). Secondly, we conducted a sensitivity analysis using an alternative case definition for fractures, which not only included fracture events identified from emergency or inpatient records but also outpatient records (Supplementary Tables 6 and 7). Otherwise we stratified our study population by median age (73 years old) to examine the interaction between age and prostateselective $\alpha$ antagonist (Supplementary Table 8). The results of additional analysis were shown in the supplements. The entire analysis was done using SAS V 9.4.

\section{CONCLUSION}

Use of prostate-selective $\alpha$ antagonists is associated with a $29 \%$ increased risk of skull fracture and $22 \%$ increased risk for femur fracture. Physicians should balance the benefits of relieving lower urinary tract symptoms with the potential risk of hypotension, falls, and fracture in this population at high risk of osteoporosis. Patients receiving prostate-selective $\alpha$ antagonists should be fully informed of fracture risk and preventive measures should be undertaken in order to reduce this risk.

\section{Author contributions}

Wei-Heng Kao and Chang-Fu Kuo had full access to all the data in the study and take responsibility for the integrity of the data and the accuracy of the data analysis.

Conceptualization: Wei-Heng Kao, Chang-Fu Kuo,

Wen-Kuan Huang.

Methodology: Wei-Heng Kao, Chang-Fu Kuo.

Software: Wei-Heng Kao, I-Jun Chou, Lai-Chu See, Meng-Jiun Chiou.

Validation: N/A.

Formal analysis: Meng-Jiun Chiou, I-Jun Chou, LaiChu See, Weiya Zhang, Jun-Te Hsu.

Investigation: Wei-Heng Kao, Chang-Fu Kuo, Meng-Jiun Chiou, Weiya Zhang, Chun-Chieh Wang.

Resources: Chang-Fu Kuo, I-Jun Chou, Michael Doherty, Hsien-Hsin Chen, Jun-Te Hsu.

Data curation: Chang-Fu Kuo, Meng-Jiun Chiou. Kuo.

Writing - original draft: Wei-Heng Kao, Chang-Fu

Writing - review and editing: Wei-Heng Kao, Chang-Fu Kuo, Ji-Hong Hong.

Visualization: Wei-Heng Kao, Chang-Fu Kuo, Meng-Jiun Chiou.

Supervision: Wei-Heng Kao, Chang-Fu Kuo.

Project administration: Wei-Heng Kao.

Funding acquisition: Chang-Fu Kuo, Ji-Hong Hong.

\section{ACKNOWLEDGMENTS}

This study is based in part on data from the National Health Insurance Research Database, which is provided by the National Health Insurance Administration, Ministry of Health and Welfare, and managed by the National Health Research Institute. The interpretation and conclusions contained herein do not represent the positions of the 
National Health Insurance Administration or the National Health Research Institute.

The authors wish to thank the Center for Big Data Analytics and Statistics (Grant CLRPG3D0043) at Chang Gung Memorial Hospital for designing the study and monitoring, analyzing, and interpreting the data.

\section{CONFLICTS OF INTEREST}

We declare that we have no conflicts of interest in the authorship or publication of this contribution.

\section{FUNDING}

The authors thank the Ministry of Science and Technology of Taiwan (project 104-2314-B-182A-047, 103-2314-B-182A-070-MY2) and Chang Gung Memorial Hospital (project CORPG3E0152, CORPG3E0142) for their financial support of this research.

\section{REFERENCES}

1. Cancer Registry Annual Report, 2012. Ministry of Health and Welfare, the Executive Yuan, Taiwan 2015. Available at: http://www.hpa.gov.tw/Bhpnet/Web/Stat/Statistics.aspx.

2. Bolla M, Collette L, Blank L, Warde P, Dubois JB, Mirimanoff RO, Storme G, Bernier J, Kuten A, Sternberg C, Mattelaer J, Lopez Torecilla J, Pfeffer JR, et al. Long-term results with immediate androgen suppression and external irradiation in patients with locally advanced prostate cancer (an EORTC study): a phase III randomised trial. Lancet. 2002; 360: 103-6.

3. Roach M 3rd, Bae K, Speight J, Wolkov HB, Rubin P, Lee RJ, Lawton C, Valicenti R, Grignon D, Pilepich MV. Short-term neoadjuvant androgen deprivation therapy and external-beam radiotherapy for locally advanced prostate cancer: long-term results of RTOG 8610. J Clin Oncol. 2008; 26: 585-91. https://doi.org/10.1200/ JCO.2007.13.9881.

4. Denham JW, Steigler A, Lamb DS, Joseph D, Turner S, Matthews J, Atkinson C, North J, Christie D, Spry NA, Tai KH, Wynne C, D'Este C. Short-term neoadjuvant androgen deprivation and radiotherapy for locally advanced prostate cancer: 10-year data from the TROG 96.01 randomised trial. Lancet Oncol. 2011; 12: 451-9. https://doi.org/10.1016/ S1470-2045(11)70063-8.

5. Saylor PJ, Smith MR. Metabolic complications of androgen deprivation therapy for prostate cancer. J Urol. 2013; 189: S34-42; discussion S3-4. https://doi.org/10.1016/j. juro.2012.11.017.

6. Kohutek ZA, Weg ES, Pei X, Shi W, Zhang Z, Kollmeier MA, Zelefsky MJ. Long-term impact of androgendeprivation therapy on cardiovascular morbidity after radiotherapy for clinically localized prostate cancer.
Urology. 2016; 87: 146-52. https://doi.org/10.1016/j. urology.2015.08.029.

7. Saad F, Olsson C, Schulman CC. Skeletal morbidity in men with prostate cancer: quality-of-life considerations throughout the continuum of care. Eur Urol. 2004; 46: 731-39; discussion 9-40. https://doi.org/10.1016/j. eururo.2004.08.016.

8. Shahinian VB, Kuo YF, Freeman JL, Goodwin JS. Risk of fracture after androgen deprivation for prostate cancer. $\mathrm{N}$ Engl J Med. 2005; 352: 154-64. https://doi.org/10.1056/ NEJMoa041943.

9. Smith MR, Lee WC, Brandman J, Wang Q, Botteman M, Pashos CL. Gonadotropin-releasing hormone agonists and fracture risk: a claims-based cohort study of men with nonmetastatic prostate cancer. J Clin Oncol. 2005; 23: 7897-903. https://doi.org/10.1200/JCO.2004.00.6908.

10. Wang A, Obertova Z, Brown C, Karunasinghe N, Bishop $\mathrm{K}$, Ferguson L, Lawrenson R. Risk of fracture in men with prostate cancer on androgen deprivation therapy: a population-based cohort study in New Zealand. BMC Cancer. 2015; 15: 837. https://doi.org/10.1186/ s12885-015-1843-3.

11. Wu CT, Yang YH, Chen PC, Chen MF, Chen WC. Androgen deprivation increases the risk of fracture in prostate cancer patients: a population-based study in Chinese patients. Osteoporos Int. 2015; 26: 2281-90. https://doi.org/10.1007/ s00198-015-3135-9.

12. Chrischilles E. Initiation of nonselective alpha1-antagonist therapy and occurrence of hypotension-related adverse events among men with benign prostatic hyperplasia: a retrospective cohort study. Clin Ther. 2001; 23: 727-43. https://doi.org/10.1016/s0149-2918(01)80022-9.

13. Tinetti ME, Kumar C. The patient who falls: "It's always a trade-off". JAMA. 2010; 303: 258-66. https://doi. org/10.1001/jama.2009.2024.

14. Dunn CJ, Matheson A, Faulds DM. Tamsulosin: a review of its pharmacology and therapeutic efficacy in the management of lower urinary tract symptoms. Drugs Aging. 2002; 19: 135-61.

15. Elhilali MM. Alfuzosin: an alpha1-receptor blocker for the treatment of lower urinary tract symptoms associated with benign prostatic hyperplasia. Expert Opin Pharmacother. 2006; 7: 583-96. https://doi.org/10.1517/14656566.7.5.583.

16. Osman NI, Chapple CR, Cruz F, Desgrandchamps F, Llorente C, Montorsi F. Silodosin : a new subtype selective alpha-1 antagonist for the treatment of lower urinary tract symptoms in patients with benign prostatic hyperplasia. Expert Opin Pharmacother. 2012; 13: 2085-96. https://doi. org/10.1517/14656566.2012.714368.

17. Bird ST, Delaney JA, Brophy JM, Etminan M, Skeldon SC, Hartzema AG. Tamsulosin treatment for benign prostatic hyperplasia and risk of severe hypotension in men aged 40-85 years in the United States: risk window analyses 
using between and within patient methodology. BMJ. 2013; 347: f6320. https://doi.org/10.1136/bmj.f6320.

18. Welk B, McArthur E, Fraser LA, Hayward J, Dixon S, Hwang YJ, Ordon M. The risk of fall and fracture with the initiation of a prostate-selective alpha antagonist: a population based cohort study. BMJ. 2015; 351: h5398. https://doi.org/10.1136/bmj.h5398.

19. Novara G, Chapple CR, Montorsi F. Individual patient data from registrational trials of silodosin in the treatment of non-neurogenic male lower urinary tract symptoms (LUTS) associated with benign prostatic hyperplasia (BPH): subgroup analyses of efficacy and safety data. BJU Int. 2015; 115: 802-14. https://doi.org/10.1111/bju.12906.

20. Resnick MJ, Lacchetti C, Bergman J, Hauke RJ, Hoffman KE, Kungel TM, Morgans AK, Penson DF. Prostate cancer survivorship care guideline: American Society of Clinical Oncology Clinical Practice Guideline endorsement. J Clin Oncol. 2015; 33: 1078-85. https://doi.org/10.1200/ JCO.2014.60.2557.

21. Souverein PC, Van Staa TP, Egberts AC, De la Rosette JJ, Cooper C, Leufkens HG. Use of alpha-blockers and the risk of hip/femur fractures. J Intern Med. 2003; 254: 548-54. https://doi.org/DOI 10.1111/j.1365-2796.2003.01227.x.

22. Hall GC, McMahon AD. Comparative study of modified release alpha-blocker exposure in elderly patients with fractures. Pharmacoepidemiol Drug Saf. 2007; 16: 901-7. https://doi.org/10.1002/pds.1402.

23. Vestergaard P, Rejnmark L, Mosekilde L. Risk of fractures associated with treatment for benign prostate hyperplasia in men. Osteoporos Int. 2011; 22: 731-7. https://doi. org/10.1007/s00198-010-1320-4.

24. Narayan P, Tewari A. A second phase III multicenter placebo controlled study of 2 dosages of modified release tamsulosin in patients with symptoms of benign prostatic hyperplasia. United States 93-01 Study Group. J Urol. 1998; 160: 1701-6.

25. Mann RD, Biswas P, Freemantle S, Pearce G, Wilton L. The pharmacovigilance of tamsulosin: event data on 12484 patients. BJU Int. 2000; 85: 446-50.

26. Norgaard M, Jensen AO, Jacobsen JB, Cetin K, Fryzek JP, Sorensen HT. Skeletal related events, bone metastasis and survival of prostate cancer: a population based cohort study in Denmark (1999 to 2007). J Urol. 2010; 184: 162-7. https://doi.org/10.1016/j.juro.2010.03.034.

27. İğdem Ş, Alço G, Ercan T, Barlan M, Ganiyusufoğlu K, Ünalan B, Turkan S, Okkan S. Insufficiency fractures after pelvic radiotherapy in patients with prostate cancer. Int J Radiat Oncol Biol Phy. 2010; 77: 818-23. https://doi. org/10.1016/j.ijrobp.2009.05.059.
28. The National Health Insurance Statistics, 2013. National Health Insurance Administration, Ministry of Health and Welfare: Taiwan, 2014. Available at: http://www.nhi.gov. tw/English/webdata/webdata.aspx?menu=11\&menu_ id=296\&WD_ID=296\&webdata_id=4645.

29. Chen CC, Chen LS, Yen MF, Chen HH, Liou HH. Geographic variation in the age- and gender-specific prevalence and incidence of epilepsy: analysis of Taiwanese National Health Insurance-based data. Epilepsia. 2012; 53: 283-90. https://doi.org/10.1111/j.1528-1167.2011.03332.x.

30. Cheng CL, Chien HC, Lee CH, Lin SJ, Yang YH. Validity of in-hospital mortality data among patients with acute myocardial infarction or stroke in National Health Insurance Research Database in Taiwan. Int J Cardiol. 2015; 201: 96-101. https://doi.org/10.1016/j.ijcard.2015.07.075.

31. Cheng CL, Kao YH, Lin SJ, Lee CH, Lai ML. Validation of the National Health Insurance Research Database with ischemic stroke cases in Taiwan. Pharmacoepidemiol Drug Saf. 2011; 20: 236-42. https://doi.org/10.1002/pds.2087.

32. Cheng CL, Lee CH, Chen PS, Li YH, Lin SJ, Yang YH. Validation of acute myocardial infarction cases in the national health insurance research database in taiwan. $\mathrm{J}$ Epidemiol. 2014; 24: 500-7.

33. Lin CC, Lai MS, Syu CY, Chang SC, Tseng FY. Accuracy of diabetes diagnosis in health insurance claims data in Taiwan. J Formos Med Assoc. 2005; 104: 157-63.

34. Kao WH, Hong JH, See LC, Yu HP, Hsu JT, Chou IJ, Chou WC, Chiou MJ, Wang CC, Kuo CF. Validity of cancer diagnosis in the National Health Insurance database compared with the linked National Cancer Registry in Taiwan. Pharmacoepidemiol Drug Saf. 2017. https://doi. org/10.1002/pds.4267.

35. Guzzo TJ, Dluzniewski P, Orosco R, Platz EA, Partin AW, Han M. Prediction of mortality after radical prostatectomy by Charlson comorbidity index. Urology. 2010; 76: 553-7. https://doi.org/10.1016/j.urology.2010.02.069.

36. Grobbee DE, Hoes AW. Confounding and indication for treatment in evaluation of drug treatment for hypertension. BMJ. 1997; 315: 1151-4.

37. Rosenbaum PR, Rubin DB. The central role of the propensity score in observational studies for causal effects. Biometrika. 1983; 70: 41-55. https://doi.org/10.1093/ biomet/70.1.41.

38. Rosenbaum PR, Rubin DB. Reducing bias in observational studies using subclassification on the propensity score. J Am Stat Assoc. 1984; 79: 516-24. https://doi.org/10.1080/ 01621459.1984 .10478078 . 